


\title{
Laparoscopy as a Diagnostic Tool in the Evaluation of Chronic Pelvic Pain in Women
}

\author{
Shailaja Chhetri \\ Shailaja Chhetri, MBBS, MD(Obstetrics and Gynecology), D.MAS
}

\begin{abstract}
Chronic pelvic pain is a common and significant disorder of women. Often the etiology of chronic pelvic pain is not clear.

Chronic pelvic pain is a common problem and presents a major challenge to health care providers because of its unclear etiology, complex natural history, and poor response to therapy. Diagnostic laparoscopy is an important tool in the evaluation of women with chronic pelvic pain.

Keywords: chronic pelvic pain, laparoscopy, pelvic adhesions, endometriosis, pelvic congestion, fibroids.
\end{abstract}

\section{INTRODUCTION}

Chronic pelvic pain is defined as a noncyclic pelvic pain of greater than six months, which is not relieved by nonnarcotic analgesics. Chronic pelvic pain has been found to be one of the most common symptoms attending gynecological outpatient department in gynecology. Prevalence of chronic pelvic pain has been reported as $3.8 \%$ in women aged $15-73$, which is higher than the prevalence of migraine (2.1\%) and similar to that of bronchial asthma (3.7\%) or back pain (4.1\%). ${ }^{1}$ In primary care practices, $39 \%$ of women complain of pelvic pain. ${ }^{2}$ Chronic pelvic pain is estimated to account for $10 \%$ of all referrals to gynecologists, it is the indication for $12 \%$ of all hysterectomies and over $40 \%$ of gynecologic laparoscopies. ${ }^{1}$ Clearly, pelvic pain is an important issue in the health care of women. Chronic pelvic pain seem to occur during reproductive years although some particular enigmatic pain syndromes may occur in young adolescents and other causes may occur after menopause and in the elderly.

Chronic pelvic pain is characterized by six common characteristics; duration of six months or longer, incomplete relief by previous treatment, pain out of proportion to tissue damage, loss of physical function at home or work, vegetative signs of depression and altered family and social roles.

Laparoscopy is a valuable clinical tool in the diagnosis of chronic pelvic pain. It can confirm a clinical impression, establish a definite diagnosis, follow the course of a disease and modify therapy accordingly.

\section{AIMS AND OBJECTIVES}

The main aim of the study is to assess the various causes of chronic pelvic pain with the help of laparoscopy as a diagnostic tool.

\section{MATERIAL AND METHODS}

A literature search was performed using Medline, Pubmed (National Library of Medicine, Bethesda, MD), search engine Google, Springerlink and Highwire press (Stanford University Libraries). In the searches of Medline, Google, Springerlink and Highwire press, the following search terms were used laparoscopy, chronic pelvic pain, pelvic adhesions, endometriosis, pelvic congestion and fibroids. Potentially relevant papers were reviewed. Selected papers were further screened for references. Criteria for selection of literature were that all were limited to human subjects who were all females, the number of cases more than 20 .

\section{COMMENTS}

The causes of chronic pelvic pain maybe somatic or nonsomatic (psychogenic). Somatic causes of chronic pelvic pain in women are commonly gynecological and the most common are pelvic adhesions, endometriosis, pelvic congestions, fibroids, adenomyosis, ovarian cysts and pelvic inflammatory disease. Nongynecological causes include irritable bowel syndrome, insterstitial cystitis, diverticulitis and mysofascial pain symptoms.

Laparoscopy, because of its availability and safety, provides a valuable tool in the evaluation of undiagnosed chronic pelvic pain. It is a simple and often definitive means of establishing the presence or absence of pelvic pathology without resorting to major abdominal surgery.

Marana et al ${ }^{4}$ performed laparoscopy in 137 patients with chronic pelvic pain and abnormal laparoscopic findings were detected in 102 (80\%) patients whereas 25 (20\%) patients had a normal laparoscopic examination. ${ }^{4}$ Kontoravdis et $\mathrm{al}^{5}$ laparoscopically assessed 1629 patients with chronic pelvic pain and detected abnormal pathology in $76 \%$ and a normal pelvic examination in $24 \%$ of the patients. Mara et al ${ }^{6}$ performed 480 laparoscopies in patients with chronic pelvic pain and pathology was detected in $82.3 \%$. Swanton et $\mathrm{al}^{7}$ did laparoscopy with conscious pain mapping in 39 women and 
identified the cause of pain in 35 (90\%) women and none in 4 (10\%) women.

Thus, the use of laparoscopy allows the detection of potentially treatable pathology not detected or detectable by other types of evaluation such as ultrasonography, imaging studies, endoscopy, and laboratory studies.

\section{LAPAROSCOPY AND PELVIC ADHESIONS}

Adhesions are among the most common organic findings noted at the time of diagnostic laparoscopy performed for the evaluation of chronic pelvic pain. They form after trauma to the visceral and parietal peritoneum and thus can be secondary to surgery, infection and endometriosis. Between 70-85\% are thought to occur after surgery. ${ }^{3}$ Cheong et al in 2006 reported that endometriosis was a typical finding in $24 \%$ of patients with chronic pelvic pain. ${ }^{8}$ Drozgyik et al in 2007 performed laparoscopy on 1061 patients with chronic pelvic pain and found that the most common cause was pelvic adhesions, which detected in $32.5 \%$ patients. ${ }^{9}$

\section{LAPAROSCOPY AND ENDOMETRIOSIS}

Sampson in 1921, defined endometriosis as "presence of ectopic endometrial tissue which possesses the histological structure and function of the uterine mucosa”, and still remains the accepted definition. Thus, endometriosis is a histological diagnosis and thus a tissue specimen is required. Thus laparoscopy is essential in the diagnosis of endometriosis, and an exploratory laparotomy is not warranted until the symptoms are extreme or laparoscopy is not available.

Endometriosis is also a common diagnosis in patients evaluated for chronic pelvic pain using diagnostic laparoscopy. The prevalence of endometriosis that was detected in laparoscopy is 20.4 to $22.3 \%$. ${ }^{6,9}$ Some studies report confirmation of suspected endometriosis during diagnostic laparoscopy in 78 to $84 \%$ of patients. ${ }^{10,11}$ Based on several randomized control trails, endometriosis appears to be responsible for chronic pelvic pain in more than half of confirmed cases. ${ }^{12}$

\section{LAPAROSCOPY AND PELVIC CONGESTION}

Pelvic congestion is associated with pelvic varicosities and it is often found in women with chronic pelvic pain in whom no other obvious cause of their pelvic pain can be found. However laparoscopy is not the recommended method for making the diagnosis of pelvic congestion syndrome and thus retrograde ovarian venography and transuterine retrograde venography is thus required. However, decreasing the intra-abdominal pressure and gradually placing the patient in reverse Trendelenburg position can demonstrate pelvic varicosities when diagnostic laparoscopy is performed. ${ }^{13}$ The prevalence of pelvic varicosities in the broad ligament is reported as $7.3 \%$. ${ }^{9}$

\section{LAPAROSCOPY AND OVARIAN CYSTS}

Ovarian cysts rarely cause chronic pelvic pain as most are hemorrhagic and corpus luteal or follicle cysts and are cyclical and are usually asymptomatic and when they do cause symptoms they are usually acute. However, ovarian cysts which are associated with endometriosis, residual ovarian syndrome or ovarian retention syndrome are associated with chronic pelvic pain. Swanton et al detected trapped ovary in $7.7 \%$ of patients undergoing laparoscopy. ${ }^{7}$

\section{LAPAROSCOPY AND FIBROIDS}

Leiomyomas are the most common tumors found in the female genital tract. Almost one of four women have palpable fibroids by the time she reaches the 4 th decade of life. The incidence of small nonpalpable tumors discovered by endovaginal ultrasonography examination or laparoscopy is even higher. Uterine fibroids have been detected in 5.2 to $8.9 \%$ in women who have undergone laparoscopy for chronic pelvic pain.,9

\section{DISCUSSION}

The results of research on chronic pelvic pain must be reviewed carefully before the data can be generalized to primary care populations. Few studies have evaluated patients with chronic pelvic pain who present to family physicians. Much of the research has been performed on well-delineated referral populations; in women who were treated by gynecologists, in women who were treated in clinics for chronic pain in general or chronic pelvic pain specifically and in women with chronic pelvic pain that persisted despite hysterectomy. Women with chronic pelvic pain responsive to simpler and less invasive treatment seldom have been participants in these studies. Chronic pelvic pain has a myriad of possible causes, most with very little correlation between clinical evidence and the extent of disease and the quality, quantity or appearance of pain. Often, the woman with chronic pelvic pain has seen several physicians, has used alternative methods of care, may have already had a hysterectomy, may have used narcotics for pain relief and usually has multiple other complaints that span several organ systems, including dyspareunia, anorgasmy, postcoital pain, disturbances in menstrual cycle, backache, nausea, malaise, diarrhea, headaches and vertigo.

Psychological evaluation is necessary in the initial evaluation and treatment of all women with chronic pelvic pain. Even when there is no suspicion of a psychological diagnosis, it helps provide the doctor with specific information about the patient that may be related to therapeutic responsiveness and prognosis, and can be used in treatment planning, however this may not always be possible.

Not all women who experience chronic pelvic pain consult their doctor and of those who do, not all are referred for hospital 
investigations let alone laparoscopy. It was noted that among 483 Oxfordshire women with chronic pelvic pain, 195 (40\%) had not sought a medical consultation, 127 (26\%) reported a past consultation and 139 (27\%) reported a consultation for pain. ${ }^{14}$

\section{CONCLUSION}

Chronic pelvic pain is a common problem and presents a major challenge to health care providers because of its unclear etiology, complex natural history, and poor response to therapy.

Laparoscopy has a major role in the gynecologist's evaluation of potential visceral causes of chronic pelvic pain. Significant judgment is required in individualizing each woman's evaluation. Missing treatable disease prolongs the patient's suffering and may even be life-threatening in rare cases. In patients who have undergone laparoscopy, reassurance derived by the procedure may lead to improvement in their symptoms. Therefore laparoscopy has an important role in the diagnosis and treatment of patients with chronic pelvic pain and should be done in all patients with chronic pelvic pain.

\section{REFERENCES}

1. Howard FM. Chronic pelvic pain. Obstet Gynecol 2003;101(3):594-611.

2. Zondervan KT, Yudkin PL, Vessey MP, Dawes MG, Barlow $\mathrm{DH}$, et al. Prevalence and incidence in primary care of chronic pelvic pain in women: Evidence from a national general practice database. Br J Obstet Gynaecol 1999;106:1149-55.

3. Vincent K. Chronic pelvic pain in women. Post Grad Med J 2009;85:24-29.
4. Marana R, Paielle FV, Muzii L, Dell’Acqua S, Mancuso S. The role of laparoscopy in the evaluation of Chronic Pelvic Pain: Minerva Ginecol 1993 Jun;45(6):281-86.

5. Kontoravdis A, Hassan E, Hassiakos D, Botsis D, KontoravdisN, Creatsas G. Laparoscopic evaluation and management of chronic pelvic pain during adolesence: Clin Exp Obstet Gynecol 1999;26(2):76-77.

6. Mara M, Fucikova Z, Kuzel D, Dohnalova A, Haakova L, et al. Laparoscopy in chronic pelvic pain-a retrospective clinical study: Ceska Gynekol 2002;67(1):38-46.

7. Swanton A, Iyer L, Reginald PW. Diagnosis, treatment and follow up of women undergoing conscious pain mapping for chronic pelvic pain: a prospective cohort study. BJOG 2006;113:79296.

8. Cheong Y, Stones WR. Management of chronic pelvic pain: evidence from randomized controlled trials. Obstet Gynecol 2006;8:32-38.

9. Drozgyik I, Vizer M, Szabo I. Significance of laparoscopy in the management of chronic pelvic pain. Eur J Obstet Gynecol Reprod Biol 2007;133(2):223-26.

10. Wood C, Kuhn R, Tsaltas J. Laparoscopic diagnosis of endometriosis. Obstet Gynecol 2002;42(3):277.

11. Richardson WS, Stefanidis D, Chang L, Earle DB, Fanelli RD. The role of laparoscopy for chronic abdominal conditions: an evidence based review. Surg Endosc 2009. Apr 9 (Epub ahead of print).

12. Fauconnier A, Chapron C. Endometriosis and pelvic pain: epidemiological evidence of the relationship and implications. Hum Reprod. Update 2005;11:595-606.

13. Beard RW, Reginald PW, Wadsworth J. Clinical features of women with chronic lower abdominal pain and pelvic congestion. Br J Obstet Gynecol 1988;98:988-92.

14. Stones RW, Price C. Health Services for women with chronic pelvic pain. J R Soc Med 2002;95:531-35. 